\title{
Perceptions of parents and religious leaders regarding minimal invasive tissue sampling to identify the cause of death in stillbirths and neonates: results from a qualitative study
}

Anam Feroz ${ }^{1 *}$ (D, Mohsina Noor Ibrahim², Elizabeth M. McClure ${ }^{5}$, Anum Shiraz Ali ${ }^{1}$, Shiyam Sunder Tikmani ${ }^{1}$, Sayyeda Reza', Zahid Abbasi', Jamal Raza², Haleema Yasmin ${ }^{3}$, Khadija Bano ${ }^{3}$, Afia Zafar ${ }^{4}$, Sameen Siddiqi ${ }^{1}$, Robert L. Goldenberg ${ }^{6}$ and Sarah Saleem ${ }^{1}$

\begin{abstract}
Background: Recently, the minimal invasive tissue sampling (MITS) procedure has been developed to support determination of the cause of death as an alternate to conventional autopsy, especially in countries where complete diagnostic autopsy is not routine. To assess the feasibility of implementation of the MITS procedure for a study to determine cause of death in premature births and stillbirths in south Asia, we explored the views and perceptions of parents and religious leaders on the acceptability of MITS.
\end{abstract}

Methods: A qualitative study was conducted at the National Institute of Child Health (NICH) hospital of Karachi, Pakistan. Focus group discussions (FGDs) were conducted with parents of newborns who visited well-baby clinics of the NICH hospital for post-natal check-ups. Key-informant interviews (KIls) were conducted with religious leaders. Data were analyzed using NVivo 10 software.

Results: A total of 13 interviews ( $F G D s=8 ; K l l s=5$ ) were conducted. Three overarching themes were identified: (I) acceptability of MITS; (II) concerns affecting the implementation of MITS; and (III) religious and cultural perspectives. Participants' acceptance of MITS was based on personal, religious, cultural and social beliefs. Parents widely recognized the need for this procedure in cases where the couple had experienced multiple stillbirths, neonatal deaths and miscarriages. Counseling of parents was considered vital to address emotional concerns of the parents and the family. Religious leaders indicated acceptability of the MITS procedure from a religious perspective and advised that respect for the deceased and consent of the guardians is mandatory when performing MITS.

Conclusions: This qualitative study provided a unique opportunity to understand the views of parents and religious leaders towards the use of MITS. Generally, MITS appears to be an acceptable method for identifying the cause of death in neonates and stillbirths, provided that the deceased is respected and buried as soon as possible without any delays and parents are counseled appropriately. Findings from this research are essential in approaching families for consent for MITS.

Keywords: Minimal invasive tissue sampling, Cause of death, Neonates, Stillbirth, Qualitative study, Parents, Perceptions, Religious leaders, Acceptability, Concerns, Counseling

\footnotetext{
* Correspondence: anam.feroz@aku.edu

${ }^{1}$ Department of Community Health Sciences, The Aga Khan University,

Stadium Road, Box 3500, Karachi, PO 74800, Pakistan

Full list of author information is available at the end of the article
}

(c) The Author(s). 2019 Open Access This article is distributed under the terms of the Creative Commons Attribution 4.0 International License (http://creativecommons.org/licenses/by/4.0/), which permits unrestricted use, distribution, and reproduction in any medium, provided you give appropriate credit to the original author(s) and the source, provide a link to the Creative Commons license, and indicate if changes were made. The Creative Commons Public Domain Dedication waiver (http://creativecommons.org/publicdomain/zero/1.0/) applies to the data made available in this article, unless otherwise stated. 


\section{Plain English summary}

Recently, the minimal invasive tissue sampling (MITS) procedure has been developed to help inform the cause of child death. Qualitative studies also suggest that MITS appears to be acceptable and technically feasible because it is non-disfiguring, less difficult, and inexpensive and can be performed quickly, thus fewer delays for the family and potential burial practices. However, such findings may not be relevant for a country like Pakistan, where $96 \%$ of the total population is Muslim. Therefore, we undertook a study to explore and understand the acceptability of MITS among parents, and religious leaders in Pakistan. Interviews were conducted with parents of newborns who visited well-baby clinics of the NICH hospital for post-natalcheck-ups and with religious leaders. Parents widely recognized the need for this procedure in cases where the couple had experienced multiple stillbirths, neonatal deaths and miscarriages. Counseling of parents was considered vital to address emotional concerns of the parents and the family. Religious leaders indicated acceptability of the MITS procedure and advised that respect for the deceased and consent of the guardians is mandatory when performing MITS. Findings from this research are essential in approaching families for consent for MITS.

\section{Background}

In low-and-middle-income countries (LMICs), verbal autopsies (VA) are the most common technique for investigating the cause of child death [1]. However, studies indicate that VA methodology is often inaccurate and has poor specificity [2]. As a consequence, there remain high uncertainties in regional and national estimates of causes of child mortality due to poor cause of death (CoD) investigations [3]. The feasibility and acceptability of complete diagnostic autopsy (CDA), the gold standard for $\mathrm{CoD}$ determination, faces noticeable challenges and acceptability issues [4]. These include resource constraints, fear of body disfigurement and cultural and religious criticisms [5]. Recently, the minimal invasive tissue sampling (MITS) procedure has been developed to help inform the CoD determination. This procedure involves body inspection and recording of basic anthropometric data, body palpation, body imaging and biopsy to obtain samples of lungs, brain, liver and other organs for histopathologic and microbiologic examination rather than extensive examination of internal organs $[4,6]$.

Preliminary findings of a multicenter research project to directly compare MITS with full-autopsy found that MITS has a good diagnostic concordance with CDA, and is an acceptable alternative to full autopsy for CoD determination [7]. Qualitative studies also suggest that MITS appears to be acceptable and technically more feasible than the CDA because it is non-disfiguring, less difficult, and inexpensive and can be performed quickly, thus fewer delays for the family and potential burial practices [8-11]. However, such findings may not be relevant for a country like Pakistan, where $96 \%$ of the total population is Muslim [12, 13]. In addition, MITS may function differently across countries with diverse health care systems. Therefore, it is important to explore parents' and religious experts' perceptions of the MITS procedure and their decision-making processes within the specific context as the procedure involves several technical, religious and cultural challenges.

To address these concerns, we undertook a study to explore and understand the acceptability of MITS among parents, and religious leaders in Pakistan [10]. This study was conducted in preparation for a larger study, the Project to Understand and Research Preterm Pregnancy Outcome in South Asia (PURPOSe). For PURPOSe, MITS will be performed on both stillbirths and preterm neonates to help determine the CoD [14]. Before the implementation of PURPOSe, there was uncertainty about how parents' would perceive MITS and whether it would be considered acceptable in places where the Muslim religion was predominant.

\section{Methods}

\section{Study design and setting}

We used an exploratory qualitative research design to collect data from the purposively sampled population as described in detail elsewhere [10]. The study was conducted at the National Institute of Child Health (NICH) hospital, because of their well-established pediatric care protocols and willingness to participate in the study. FGDs were conducted at the outpatient department (OPD) and well-baby clinics of the NICH hospital, while KIIs were conducted at the respective locations of key-informants.

\section{Study participants}

Parents of newborns who were visiting the OPD and well-baby clinics of NICH hospital for regular growth monitoring, post-natalcheck-ups and vaccinations were purposively sampled for focus group discussions (FGDs). Considering the cultural and ethical sensitivity, the study excluded parents and/or families that experienced a recent neonatal death/stillbirth or who were in-patients. Religious leaders, including Sunni Ulemas and Shia Muftis were purposively sampled for key-informant interviews (KIIs). In addition, we interviewed health care providers with results presented elsewhere.

\section{Interview guide}

Separate semi-structured interview guides for FGDs and KIIs explored participants' views and attitudes towards MITS, with focus on the cultural and religious perspectives, perceived benefits, concerns affecting implementation and 
factors facilitating the implementation. Before beginning the interview, the qualitative researchers first described an overview of autopsy and the MITS procedure (Table 1).

\section{Data collection}

A free flow of information was encouraged, using probes from these discussions to obtain parents' perception of the MITS procedure. Interviews were conducted face-to-face in Urdu or English according to the participants' preferences and were audio recorded with consent from study participants. Detailed field notes were also taken during each interview to capture non-verbal language and cues. KIIs lasted between 20 and $40 \mathrm{~min}$; FGDs lasted $30 \mathrm{~min}$ to $1 \mathrm{~h}$ and consisted of five participants per group.

\section{Analysis}

Study data were analyzed using thematic analysis facilitated by NVivo version 10 (QSR International, Pty Ltd) software. Data were collected and analyzed through an iterative process and data collection was ceased once saturation was achieved. First, the audio recordings were transcribed into English and then the transcriptions were uploaded in the software. Transcripts were read several times to develop an interpretation of the participants' perception towards MITS. Focus groups and KIIs were coded as two data sets. Two investigators coded a subset of transcripts independently using separate NVivo files which were then combined to match codes, and agreement was sought on a coding framework. The remaining transcripts were then independently coded according to the framework. Codes were formulated inductively from the transcripts. Coding discrepancies were discussed and resolved to reduce researcher's bias. Codes were then analyzed under major themes. Semi-structured interview guides were used to outline major themes. Final overarching themes were discussed and reviewed by both investigators. To ensure the credibility of the research, study data were triangulated by the data sources

Table 1 Overview of open autopsy and MITS procedure

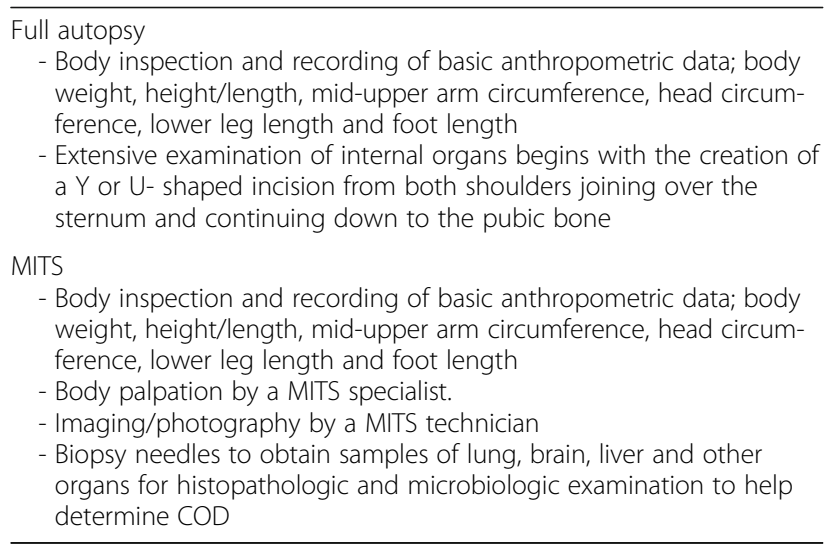

(mothers, fathers, religious leaders) and data collection methods (FGDs and KIIs), to compare alternative perspectives and assess any inconsistencies.

\section{Ethical considerations}

Ethical approval for this study was obtained from the $\mathrm{NICH}$ hospital [11/2018] and the Aga Khan University Ethical Review Committee (AKU-ERC) - [5358-CHS-ERC- 18]. Written informed consent was provided by all study participants. Informed consent included permission to audio record the interviews and use anonymized quotes.

\section{Results}

In this qualitative study, eight FGDs and five KIIs were conducted with a variety of stakeholders (parents and religious leaders), between July 2018 and August 2018, to ascertain the acceptability of MITS to identify CoD among stillbirths and neonates (Table 2). The demographic information for the FGD participants is illustrated in Table 3. All the study participants $(n=45)$ who were approached by the study team agreed to participate in the study. All the respondents were Muslim.

Based on the data collection and thematic analyses, three overarching themes were identified (Table 4): (I) acceptability of MITS; (II) concerns affecting the implementation of MITS; and (III) religious and cultural perspectives of the KIIs. These themes are presented below with illustrative quotes

\section{Focus group discussions with parents Acceptability of MITS}

Likely uptake in recurrent pregnancy loss Generally, most parents showed a positive attitude towards the MITS procedure. Parents widely recognized that there likely will be uptake for MITS procedure in cases where parents have experienced multiple neonatal deaths, stillbirths or miscarriages.

"I know a lady who has lost her four or five children and she still does not know the cause of death. In

Table 2 Study Participants

\begin{tabular}{|c|c|}
\hline Focus Group Discussions with Parents & Total FGDs $=8 ; n=40$ \\
\hline $\begin{array}{l}\text { Mothers of newborns who were visiting OPD } \\
\text { and well-baby clinics of NICH hospital for } \\
\text { regular post-natal check-ups }\end{array}$ & $\begin{array}{l}4 \text { groups } \\
\text { (5 participants in } \\
\text { each group) }\end{array}$ \\
\hline $\begin{array}{l}\text { Fathers of newborns who were visiting OPD } \\
\text { and well-baby clinics of NICH hospital for } \\
\text { regular post-natal check-ups }\end{array}$ & $\begin{array}{l}4 \text { groups } \\
\text { ( } 5 \text { participants in } \\
\text { each group) }\end{array}$ \\
\hline Key Informant Interviews with Religious Leaders & Total Klls $=5 ; n=5$ \\
\hline Sunni Ulemas & 3 Klls \\
\hline Shia Muftis & $2 \mathrm{Klls}$ \\
\hline
\end{tabular}


Table 3 Demographic information for the FGD study participants

\begin{tabular}{|c|c|c|c|c|c|c|}
\hline Pseudonym & Age & Gender & Socio-economic status & Religion & Education & Employment \\
\hline \multicolumn{7}{|c|}{ FGDs with mothers of new-borns who were visiting OPD and well-baby clinics of NICH hospital for regular post-natal check-ups } \\
\hline \multicolumn{7}{|l|}{ FGD 01} \\
\hline FGD01-W01 & 32 & F & Lower & Muslim & Grade 5 & Unemployed \\
\hline FGD01-W02 & 24 & $\mathrm{~F}$ & Lower & Muslim & None & Unemployed \\
\hline FGD01-W03 & 33 & $F$ & Lower-middle & Muslim & None & Unemployed \\
\hline FGD01-W04 & 27 & $\mathrm{~F}$ & Lower & Muslim & None & Unemployed \\
\hline FGD01-W05 & 26 & F & Lower & Muslim & Grade 8 & Unemployed \\
\hline \multicolumn{7}{|l|}{ FGD 02} \\
\hline FGD02-W01 & 27 & $\mathrm{~F}$ & Lower-middle & Muslim & None & Unemployed \\
\hline FGD02-W02 & 38 & F & Lower & Muslim & None & Unemployed \\
\hline FGD02-W03 & 42 & F & Lower & Muslim & Grade 6 & Unemployed \\
\hline FGD02-W04 & 26 & F & Lower-middle & Muslim & None & Unemployed \\
\hline FGD02-W05 & 36 & $\mathrm{~F}$ & Lower & Muslim & Grade 8 & Unemployed \\
\hline \multicolumn{7}{|l|}{ FGD 03} \\
\hline FGD03-W01 & 29 & $\mathrm{~F}$ & Lower & Muslim & None & Unemployed \\
\hline FGD03-W02 & 32 & $\mathrm{~F}$ & Lower & Muslim & None & Unemployed \\
\hline FGD03-W03 & 22 & $\mathrm{~F}$ & Lower-middle & Muslim & Grade 2 & Unemployed \\
\hline FGD03-W04 & 34 & $F$ & Lower & Muslim & None & Unemployed \\
\hline FGD03-W05 & 37 & $\mathrm{~F}$ & Lower & Muslim & None & Unemployed \\
\hline \multicolumn{7}{|l|}{ FGD 04} \\
\hline FGD04-W01 & 19 & F & Lower & Muslim & None & Unemployed \\
\hline FGD04-W02 & 38 & $\mathrm{~F}$ & Lower & Muslim & None & Unemployed \\
\hline FGD04-W03 & 23 & $\mathrm{~F}$ & Lower-middle & Muslim & Grade 3 & Unemployed \\
\hline FGD04-W04 & 35 & $\mathrm{~F}$ & Lower-middle & Muslim & Diploma & Employed \\
\hline FGD04-W05 & 28 & $\mathrm{~F}$ & Lower & Muslim & None & Unemployed \\
\hline
\end{tabular}

FGDs with fathers of new-borns who were visiting OPD and well-baby clinics of NICH hospital for regular post-natal check-ups FGD 05

\begin{tabular}{|c|c|c|c|c|c|c|}
\hline FGD05-M01 & 39 & M & Lower & Muslim & None & Employed \\
\hline FGD05-M02 & 45 & M & Lower & Muslim & Grade 7 & Employed \\
\hline FGD05-M03 & 32 & M & Lower-middle & Muslim & None & Employed \\
\hline FGD05-M04 & 31 & M & Lower & Muslim & None & Employed \\
\hline FGD05-M05 & 48 & M & Lower & Muslim & None & Employed \\
\hline \multicolumn{7}{|l|}{ GD 06} \\
\hline FGD06-M01 & 36 & M & Lower & Muslim & None & Employed \\
\hline FGD06-M02 & 29 & M & Lower & Muslim & Grade 3 & Employed \\
\hline FGD06-M03 & 34 & M & Lower & Muslim & None & Employed \\
\hline FGD06-M04 & 47 & M & Lower & Muslim & None & Employed \\
\hline FGD06-M05 & 33 & M & Lower-middle & Muslim & None & Employed \\
\hline \multicolumn{7}{|l|}{07} \\
\hline FGD07-M01 & 27 & M & Lower & Muslim & None & Employed \\
\hline FGD07-M02 & 36 & M & Lower & Muslim & Grade 1 & Employed \\
\hline FGD07-M03 & 42 & M & Lower & Muslim & None & Employed \\
\hline FGD07-M04 & 28 & M & Lower & Muslim & None & Employed \\
\hline FGD07-M05 & 41 & M & Lower & Muslim & None & Employed \\
\hline
\end{tabular}


Table 3 Demographic information for the FGD study participants (Continued)

\begin{tabular}{|c|c|c|c|c|c|c|}
\hline Pseudonym & Age & Gender & Socio-economic status & Religion & Education & Employment \\
\hline \multicolumn{7}{|l|}{ FGD 08} \\
\hline FGD08-M01 & 26 & M & Lower & Muslim & None & Employed \\
\hline FGD08-M02 & 39 & M & Lower-middle & Muslim & None & Employed \\
\hline FGD08-M03 & 47 & M & Lower & Muslim & None & Employed \\
\hline FGD08-M04 & 32 & M & Lower & Muslim & Grade 6 & Employed \\
\hline FGD08-M05 & 38 & M & Lower & Muslim & None & Employed \\
\hline
\end{tabular}

such cases, a mother will opt for MITS to know the cause of her child's death." (Mother, FGD)

Most parents expressed willingness to consent for MITS as it would inform them about the possible reasons that led to the child's death. Parents verbalized that they would want to know the CoD so that remedial actions could be taken to prevent deaths in subsequent pregnancies.

"Many parents would give permission for this procedure as it would help them prevent further deaths in subsequent pregnancies." (Mother, FGD)

Willing to know the cause of death of a loved one Among most mothers, the potential acceptability of the MITS procedure was associated with their willingness to know the CoD of a loved one. One mother mentioned that a few years back she experienced the sudden death of her child and would have requested this procedure to

Table 4 Themes and sub-themes

\begin{tabular}{|c|c|}
\hline Theme & Sub-theme \\
\hline \multirow[t]{7}{*}{ Acceptability of MITS } & $\begin{array}{l}\text { Likely uptake in recurrent pregnancy } \\
\text { loss }\end{array}$ \\
\hline & $\begin{array}{l}\text { Willing to know the cause of death of } \\
\text { a loved one }\end{array}$ \\
\hline & Doesn't bring the dead back to life \\
\hline & Perceived societal benefit \\
\hline & $\begin{array}{l}\text { Socio-demographic factors influencing } \\
\text { the acceptance of procedure }\end{array}$ \\
\hline & Fear of unexpected medical findings \\
\hline & Counseling for MITS \\
\hline \multirow{4}{*}{$\begin{array}{l}\text { Concerns affecting } \\
\text { implementation of MITS } \\
\text { procedure }\end{array}$} & State of mind around death \\
\hline & Disrespect for the deceased \\
\hline & Possible delays to funeral \\
\hline & Consent and decision making \\
\hline \multirow{3}{*}{$\begin{array}{l}\text { Religious and cultural } \\
\text { perspective }\end{array}$} & Religious permissibility \\
\hline & $\begin{array}{l}\text { Compelling justification for performing } \\
\text { the procedure }\end{array}$ \\
\hline & Seeking permission of the guardian \\
\hline
\end{tabular}

understand what went wrong at the ninth month of pregnancy.

"My first baby was born dead... Four days before the delivery, I got to know that the baby has died... I wanted to know what happened... I would have gone for MITS because my baby was perfectly fine for the whole nine months. I came for the checkups, doctors told me my baby is doing perfectly fine, and they gave me an expected date of delivery. Then something wrong happened suddenly. At least I could have explored what went wrong." (Mother, FGD)

Fathers discussed that most people in the city are not cognizant about the methods that can help identify the cause of child death. They believed that most parents would agree for the procedure if they would be informed that non-invasive option is available.

"There are many people here in cities that are not aware of what can be done to identify the cause of death. If you will tell them, they will allow you to find out what went wrong." (Father, FGD)

Doesn't bring the dead back to life A few mothers verbalized that after death there was no hope and nothing which could help the deceased child. Therefore, most parents would not be willing to undertake any such procedure. Several fathers also mentioned that parents would not provide permission as MITS could not bring back their deceased child, as illustrated in the following quote:

"This would be a very emotional situation for the mother, because she has lost her child keeping him/ her in her womb for months. She will never agree as it would not help her bring back the deceased child. If I was at her place I would have never agreed for this." (Mother, FGD)

Perceived societal benefit Most parents reported that they would be willing to consent for MITS if it could 
save the life of someone else's child in the future. For several mothers, the procedure was seen as beneficial for the community and society in general as it has the potential to provide better knowledge about the diseases and treatments.

"If my child can save someone else's child then yes definitely, this should be done." (Mother, FGD)

Socio-demographic factors influencing the acceptance of the procedure Most parents verbalized that acceptance highly depends on one's religious beliefs. Several parents mentioned that they would like to know the religious interpretation of these procedures and expressed a desire to consult religious leaders to know whether such procedures are permissible in Islam.

"This depends on what religious people have to say, because we will have to see if Islam gives permission for this. If Islam permits then people will also support you... if it is in our good faith, then this should be done." (Father, FGD)

In addition to religious beliefs, parents believed that education and socio-economic status (SES) are also important factors that determine acceptance for MITS procedure. Some mothers indicated that unfortunately most child deaths occur in impoverished families where death is viewed as nature's decision. A few fathers reported that only educated families and parents would understand the need for such procedures.

"In my opinion, if the family has an educated background, they would show willingness for MITS procedure. If they do not have that capacity, they will consider death as Allah's will." (Father, FGD)

Fear of unexpected medical findings $A$ few mothers believed that most women would not consent because of the fear of unexpected medical findings. Mothers verbalized that for most women, it would be really difficult to agree for this procedure because if something unexpected is discovered during the procedure, the husband and in-laws would blame them. Mothers expressed acute anxiety with the thought that their marriage could end or their husbands would opt for a second marriage.

"For a mother, there's a fear that if she would go for the tests and something unexpected comes out, the husband and in-laws will trouble her."

(Mother in an FGD)
Counseling for MITS Mothers acknowledged that effective counseling is crucial for the acceptability of the MITS procedure. Parents and religious leaders widely recognized the role of trained counselors who are mindful of common cultural and religious concerns when discussing MITS with parents/families. Moreover, parents emphasized that religious leaders should be part of the counseling team as they would help address religious concerns. A few parents suggested that the hospital team should first communicate with the elders of the family as they can better convince the parents of the need to determine the $\mathrm{CoD}$ of the deceased child.

"A parent is so emotional at that time ...I am saying this because I have been through this traumatic situation. If somebody could convince him for this procedure, that could be his parents (grandparent of the deceased child)." (Father, FGD)

\section{Concerns affecting implementation of MITS procedure}

State of mind around death Parents verbalized that the family's state of mind around child death is an important concern affecting acceptance and implementation of MITS. Mothers reported that it will be challenging to approach a family for MITS immediately after the death as they will be experiencing acute grief. Most mothers suggested that physicians should be very empathetic and gentle with the parents of the deceased and should be able to respond to their concerns.

"This would be a very emotional situation for the mother as she has lost her child after keeping him in her womb for months. She will never agree. If I was at her place, I would have never agreed for this."

(Mother, FGD)

Disrespect for the deceased Despite the MITS procedure potentially being religiously acceptable, some parents stated that they would decline consent for this procedure since it is considered disrespectful in their culture. Moreover, they believed that death was meant to happen as it was Allah's will and therefore they would not want to know the CoD.

"See this is very difficult for parents. They would not allow for this procedure even if it is religiously acceptable. Many parents would find it disrespectful." (Father, FGD)

Possible delays to funeral A few parents believed that the MITS procedure could delay the funeral. Fathers 
indicated that especially families from distant places are likely to decline the procedure as they would like to go back to their villages soon after the death to carry out religious customs surrounding burial. Mothers mentioned that parents would not be willing for any such procedure and would want to bury their deceased child as soon as possible without any delays. As one mother in FGD explained:

"If Allah has given me the child and then took it back from me, I would want to bury my child as soon as possible." (Mother, FGD)

Consent and decision making Social relationships were another factor that affects the consent and decision-making process for MITS. Most mothers verbalized that in a patriarchal marriage, females have little control or influence over such situations and the husbands are authorized to make final decisions about such procedures. In some cases, grandparents are given authority for such decisions as they are perceived to have a mature perspective. Most mothers mentioned that even if they provided consent for the MITS procedure, the society and family members would humiliate them and impede their participation, as illustrated by the following quote:

"Even if a mother would agree for MITS, elders of the family would say she is doing this kind of thing with her child." (Mother, FGD)

\section{Key informant interviews Religious and cultural perspectives}

Religious permissibility Most religious leaders expressed concerns related to the extent to which the deceased would be touched and affected. The religious key-informants mentioned that the dead body should be examined very carefully during the procedure so that it has minimal damage. One religious leader suggested that the procedure should be rigorously followed to avoid any further mutilation.

"If you can do the samplings from the body with minimum damage, then there is no restriction from Shariah. However, on the conditions that the respect of the body is considered and not going any further of what you basically require." (Religious Leader, KII)

Compelling justification for performing the procedure Most of the religious leaders believed that MITS would only be permissible if there is a compelling justification to perform the procedure. All religious leaders noted that without any persuasive reason, mutilating a dead body is forbidden in Islam and that the dead body should be given utmost respect and dignity. Many religious leaders voiced their opinion that the dead body could still feel pain and therefore, respect for the deceased is mandatory.

"According to Shariah of Prophet Mohammed (P.B.U.H), the respect for the body after death is the same as it is during life... Respect for the body after death is slightly greater, and touching a living or dead body without any valid reason is strongly prohibited." (Religious leader, KII)

Seeking permission of the guardian Religious leaders emphasized that obtaining the permission of the guardian before performing the procedure is mandatory. The respect for the body is mandatory in Islam and the guardian bears the responsibility of ensuring that the deceased is fully respected. The religious leaders recommended that before initiating any such procedure, the first step would be to take consent from the guardian, as illustrated by the following quote:

"The first law is the respect of the body, and the rightful heir bears the responsibility of the respect of body... If we had to do some research for which we require touching or looking at the dead body to find out what went wrong, then the first step is to seek permission of the rightful heir." (Religious leader, KII)

\section{Discussion}

This study identified the factors influencing the acceptability of MITS among Muslim parents in Pakistan. Generally, MITS appears to be an acceptable method for identifying the $\mathrm{CoD}$ in neonates and stillbirths. According to a previous study, the procedure has received resistance from the Muslim communities [13]. However similar findings were reported in a socio-behavioral study conducted in Africa and South Asia to understand the feasibility and hypothetical acceptability of MITS. The research found that majority of the Muslim participants interviewed would be willing to know the CoD (78\%) and would accept MITS (70\%) [15]. Our study further informs that the factors influencing the acceptability of MITS are complex and multifaceted in the context of Pakistan as most of the population is Muslim. Participants' insights regarding the perceived acceptability of MITS were based on personal, religious, cultural and social beliefs. 
The majority of the parents widely recognized the need for this procedure for couples who have experienced multiple neonatal deaths, stillbirths and miscarriages. They also articulated that the parents who have experienced loss of a baby would grant permission for MITS to establish the cause of child's death. Interestingly, these viewpoints align with those in the Lewis et al. study where FGD participants indicated acceptance for the less invasive autopsy if the family had experienced multiple fetal losses [16]. Most importantly, the study suggested that the high hypothetical acceptability of MITS was due to parent's own willingness to know the cause of child death. With reference to the acceptance of MITS, counseling for the parents was considered vital to address emotional concerns. Previous studies have similarly reported that providing complete information and support to the families through counseling is crucial for the acceptability of MITS among parents [4, 9]. Another enabling factor for the uptake of MITS was the procedure's potential to benefit the society and community at large. Finally, parents' educational status was reported as a likely contributor to the acceptance for MITS procedure as educated families are believed to be better able to understand the need for such procedures.

A number of concerns affecting the implementation of the MITS procedure were identified in the study. These included parent's state of mind around the time of the death, possible delays to the funeral, perceived disrespect of the deceased and fear of unexpected medical findings, consistent with the previous research findings [16].

Social relationships can play a major role in the uptake of MITS. Previous studies have also recognized that social relations, power and gender dynamics are crucial to acceptance for MITS [17]. While most participants reported that mothers-in-law and elders are the decision-makers, in some cases, fathers are the primary decision-makers over matters involving the deceased child. It can be argued that women's lack of decision-making power could affect the uptake of MITS. Therefore, it is necessary to advocate for the rights of mothers and wives to consent for MITS [17]. Similar to our study, a study in Bangladesh suggested that women would be unable to refuse MITS if their partners agreed and therefore, unequal power relationships could coerce participants to agree against their will. This study concludes that investigators should be aware of potential harms and should address them through corrective actions [5].

Religious and cultural perspectives emerged as the main theme, which was perceived both as a facilitator and a barrier towards the acceptability of MITS. Contrary to preconceptions on this subject, Muslim leaders such as Shia Muftis and Sunni Ulemas, from the two major sects of Islam, indicated high acceptability for the MITS procedure. To address the religious concerns, ensuring that there is respect for the deceased, no mutilation to the body and permission of the guardian when there was reasonable justification were the main factors to increase acceptability of MITS. Most parents argued that religious leaders, in particular Muslim leaders, should determine the acceptability of MITS, similar to reports from studies in other Muslim societies [5].

Several parents believed that the body is sacred after death and should not be disrespected by any means. They believed that the deceased should be buried as soon as possible after death, with all burial rituals similar to other studies [9]. This indicates the need for ensuring that the trained counselors and health care providers are mindful of the common religious and sociocultural beliefs when discussing MITS with parents/families.

While the findings of this study are promising for the potential uptake of MITS for CoD determination, they remain theoretical and will be validated with data regarding the acceptance during the implementation study. Yet, there are some study findings which have influenced the manner in which consent is obtained in the main study (PURPOSe). These include first, that the primary physician team should introduce new members of the MITS team to the patient/family; counsellors should seek consent, primarily from parents in-person and in a quiet and private setting; counselors should understand MITS procedure from a religious perspective; and counsellors should describe the MITS procedure like a biopsy technique. The major limitation of this research is that the acceptance of MITS was explored with the parents in a hypothetical situation.

\section{Conclusion}

This qualitative study provided an opportunity to explore the views of parents and religious leaders towards MITS. In general, parents viewed the MITS procedure positively when it is performed with respect of the deceased. Findings from this research will be essential in providing appropriate counseling and consent for MITS. Ultimately to understand the feasibility and acceptability of MITS in Pakistan, close follow-up of study participants is needed to understand the consent and decision-making process, and explore factors that could affect the acceptance for MITS. This exploratory qualitative study could also be replicated in settingswhere preliminary feasibility and acceptability of MITS is not ascertained among parents, families and religious leaders.

\section{Abbreviations}

CDA: Complete Diagnostic Autopsy; CoD: Cause of Death; ERC: Ethical Review Committee; FGDs: Focus Group Discussions; KIls: Key-informant Interviews; LMIC: Low-and-middle-income-countries; MITS: Minimal Invasive Tissue Sampling; OPD: Outpatient Department; VA: Verbal Autopsies 


\section{Funding}

This study was conducted in preparation for a larger study, the Project to Understand and Research Preterm Pregnancy Outcome in South Asia (PURPOSe). The main study is funded by the Bill \& Melinda Gates Foundation.

\section{Availability of data and materials}

The datasets used and/or analysed during the current study are available from the corresponding author on reasonable request.

\section{Authors' contributions}

SS, AF and SST conceptualized and designed the study. AF, ASA, SR, ZA and MNI oversaw data collection. AF and ASA conducted data analysis. AF prepared the first draft of the manuscript. RLG, EMM and SS reviewed the manuscript several times and provided critical feedback. All authors (AF, MNI, EMM, ASA, SST, SR, ZA, JR, HY, KB, AZ, RLG, SS, SSQ) read and approved the final version of the manuscript.

\section{Ethics approval and consent to participate}

Ethical approval for this study was obtained from the NICH hospital [11/ 2018] and Aga Khan University Ethical Review Committee (AKU-ERC) - [5358CHS-ERC- 18]. Written informed consent was provided by all study participants. Informed consent included permission to audio record the interviews and use anonymized quotes. Voluntary participation and the right to ask any questions and to decline participation at any time were emphasized during the data collection.

\section{Consent for publication}

Written informed consent for publication was obtained.

\section{Competing interests}

The authors declare that they have no competing interests.

\section{Publisher's Note}

Springer Nature remains neutral with regard to jurisdictional claims in published maps and institutional affiliations.

\section{Author details}

'Department of Community Health Sciences, The Aga Khan University, Stadium Road, Box 3500, Karachi, PO 74800, Pakistan. ${ }^{2}$ National Institute of Child Health, Karachi, Pakistan. ${ }^{3}$ Jinnah Post-graduate Medical Center, Department of Obstetrics and Gynecology-Department of Pathology and Laboratory Medicine, Karachi, Pakistan. ${ }^{4}$ Department of Pathology \& Laboratory Medicine, The Aga Khan University, Stadium Road, Box 3500 Karachi, PO 74800, Pakistan. ${ }^{5}$ RTI International, Durham, USA. ${ }^{6}$ Department of Obstetrics and Gynecology, Columbia University, New York, USA.

Received: 16 November 2018 Accepted: 1 May 2019

Published online: 10 May 2019

\section{References}

1. Leitao J, Chandramohan D, Byass P, Jakob R, Bundhamcharoen K, Choprapawon $\mathrm{C}$, et al. Revising the $\mathrm{WHO}$ verbal autopsy instrument to facilitate routine cause-of-death monitoring. Glob Health Action. 2013;6(1): 21518.

2. Rudan I, O'Brien KL, Nair H, Liu L, Theodoratou E, Qazi S, et al. Epidemiology and etiology of childhood pneumonia in 2010: estimates of incidence, severe morbidity, mortality, underlying risk factors and causative pathogens for 192 countries. J Glob Health. 2013;3(1).

3. Abubakar I, Tillmann T, Banerjee A. Global, regional, and national age-sex specific all-cause and cause-specific mortality for 240 causes of death, 19902013: a systematic analysis for the global burden of disease study 2013. Lancet. 2015;385(9963):117-71.

4. Maixenchs M, Anselmo R, Sanz A, Castillo P, Macete E, Carrilho C, et al. Healthcare providers' views and perceptions on post-mortem procedures for cause of death determination in southern Mozambique. PLoS One. 2018; 13(7):e0200058.

5. Gurley ES, Parveen S, Islam MS, Hossain MJ, Nahar N, Homaira N, et al. Family and community concerns about post-mortem needle biopsies in a Muslim society. BMC medical ethics. 2011;12(1):10.
6. Kang X, Cos T, Guizani M, Cannie MM, Segers V, Jani JC. Parental acceptance of minimally invasive fetal and neonatal autopsy compared with conventional autopsy. Prenat Diagn. 2014;34(11):1106-10.

7. Castillo P, Martínez MJ, Ussene E, Jordao D, Lovane L, Ismail MR, et al. Validity of a minimally invasive autopsy for cause of death determination in adults in Mozambique: an observational study. PLoS Med. 2016;13(11): e1002171.

8. Patton MQ. Enhancing the quality and credibility of qualitative analysis. Health Serv Res. 1999;34(5 Pt 2):1189.

9. Meaney S, Gallagher S, Lutomski JE, O'donoghue K. Parental decision making around perinatal autopsy: a qualitative investigation. Health Expect. 2015;18(6):3160-71.

10. Feroz A, Ibrahim MN, Tikmani SS, Reza S, Abbasi Z, Raza J, et al. Perceptions of parents and healthcare professionals regarding minimal invasive tissue sampling to identify the cause of death in stillbirths and neonates: a qualitative study protocol. Reprod Health. 2018;15(1):179.

11. Lewis C, Hill M, Arthurs OJ, Hutchinson JC, Chitty LS, Sebire N. Health professionals' and coroners' views on less invasive perinatal and paediatric autopsy: a qualitative study. Arch Dis Child. 2018;103(6):572-8.

12. CHaMPSC N. Assessment of community perceptions and the feasibility of conducting child mortality and pregnancy surveillance. Child health and mortality prevention surveillance (CHAMPS) network; 2016.

13. Cassum LA. Refusal to autopsy: a societal practice in Pakistan context. J Clin Res Bioethics. 2014;5(5).

14. McClure EM, Saleem S, Goudar SS, Dhaded S, Guruprasad G, Kumar Y, et al. The project to understand and research preterm pregnancy outcomes and stillbirths in South Asia (PURPOSe): a protocol of a prospective, cohort study of causes of mortality among preterm births and stillbirths. Reprod Health. 2018;15(1):89.

15. Maixenchs M, Anselmo R, Zielinski-Gutiérrez E, Odhiambo FO, Akello C, Ondire $M$, et al. Willingness to know the cause of death and hypothetical acceptability of the minimally invasive autopsy in six diverse African and Asian settings: a mixed methods socio-behavioural study. PLoS Med. 2016; 13(11):e1002172.

16. Lewis C, Latif Z, Hill M, Riddington M, Lakhanpaul M, Arthurs OJ, et al. "We might get a lot more families who will agree": Muslim and Jewish perspectives on less invasive perinatal and paediatric autopsy. PLoS One. 2018;13(8):e0202023

17. Ngwenya N, Coplan D, Nzenze S, Myburgh N, Madhi S. Community acceptability of minimally invasive autopsy (MIA) in children under five years of age in Soweto, South Africa. Anthropology Southern Africa. 2017; 40(2):108-21.

Ready to submit your research? Choose BMC and benefit from:

- fast, convenient online submission

- thorough peer review by experienced researchers in your field

- rapid publication on acceptance

- support for research data, including large and complex data types

- gold Open Access which fosters wider collaboration and increased citations

- maximum visibility for your research: over $100 \mathrm{M}$ website views per year

At $\mathrm{BMC}$, research is always in progress.

Learn more biomedcentral.com/submissions 